\title{
ANÁLISIS DE FORMULACIONES APROXIMADAS DE IMPEDANCIA INTERNA PARA EL ESTUDIO DE TRANSITORIOS DE ALTA FRECUENCIA EN CABLES SUBTERRÁNEOS DE ALTA TENSIÓN
}

\author{
José Miguel García-Guzmán¹, Jacinto Torres-Jiménez², Francisco Javier Ortega-Herrera², \\ Alfonso Lozano-Luna ${ }^{3}$ \\ ${ }^{1}$ Docente investigador en la Coordinación de Ingeniería Electromecánica. Correo electrónico: migarcia@itesi.edu.mx \\ 2 Docente investigador, Posgrado en Ingeniería Eléctrica. \\ ${ }^{3}$ Docente investigador, Coordinación de Ingeniería Electromecánica. \\ Instituto Tecnológico Superior de Irapuato. México.
}

Recibido: 12 de agosto del 2013. Aprobado: 10 de octubre del 2013.

Cómo citar este artículo: J. M. García-Guzmán, J. Torres-Jiménez, F. J. Ortega-Herrera y A. Lozano-Luna, "Análisis de formulaciones aproximadas de impedancia interna para el estudio de transitorios de alta frecuencia en cables subterráneos de alta tensión". Ingeniería Solidaria, Vol. 9, №. 16, pp. 9-23, Dic., 2013.

Resumen. En este artículo se presenta un análisis de las formulaciones aproximadas de impedancia interna para el estudio de transitorios de alta frecuencia en cables subterráneos de alta tensión. El análisis se desarrolla en el dominio de la frecuencia y la conversión al dominio del tiempo se realiza utilizando la transformada rápida de Fourier. El cable se modela como una línea de transmisión multiconductora, utilizando análisis modal y la teoría de redes de dos puertos. Las formulaciones aproximadas de impedancia interna que se analizan son las propuestas por Gary y Wedepohl-Wilcox, las cuales son utilizadas en el modelado de líneas de transmisión aéreas y subterráneas, respectivamente. Para efectuar el análisis se realiza una comparación de las formulaciones aproximadas con el modelo exacto de impedancia interna y se determina el error relativo de cada formulación para los valores reales como para los valores imaginarios. Los resultados muestran que la formulación aproximada propuesta por Gary para el modelado de líneas de transmisión aéreas se puede utilizar en cables subterráneos para analizar fenómenos transitorios electromagnéticos de alta frecuencia con resultados aceptables. Para realizar el estudio comparativo de las formulas aproximadas de impedancia interna se propone una serie de casos de estudios en los cuales se determina la magnitud del voltaje transitorio, el tiempo de viaje y la atenuación de la onda en líneas monofásicas subterráneas utilizando diferentes longitudes.

Palabras clave: cables subterráneos, error relativo, formulaciones aproximadas, impedancia interna, transitorios de alta frecuencia.

\section{Analysis of Approximate Formulas of Internal IMPEDANCE FOR THE STUDY OF HIGH-FREQUENCY Transients in High-voltage Underground Cables}

Abstract. This study presents an analysis of approximate formulas of internal impedance for the study of high-frequency transients in high-voltage underground cables. The analysis is conducted in the frequency domain, and converted to the time domain performed using a Fast Fourier Transform. The cable is modeled as a multiconductor transmission line, using modal analysis and two-port network theory. The approximate internal impedance formulas analyzed are those proposed by Gary and WedepohlWilcox, used in modeling overhead and underground transmission lines, respectively. To conduct the analysis, formulas are compared with the exact model of internal impedance and the relative error of each formula is determined for real and imaginary values. The results show that the approximate formula proposed by Gary for modeling overhead transmission lines can be used to analyze high-frequency electromagnetic transient phenomena in underground cables with acceptable results. In order to carry out a comparative study of the approximate formulas of internal impedance, a series of case studies are presented, in which the magnitude of the transient voltage, the transit time and wave attenuation are determined in different lengths of single-phase underground line.

Keywords: underground cables, relative error, approximate formulas, internal impedance, high-frequency transients.

\section{ANÁlise DE FORMULAÇões APROXIMADAS DE IMPEDÂNCIA INTERNA PARA O ESTUDO DE TRANSITÓRIOS DE ALTA FREQUÊNCIA EM CABOS SUBTERRÂNEOS DE ALTA TENSÃO}

Resumo. Neste artigo, apresenta-se uma análise das formulações aproximadas de impedância interna para o estudo de transitórios de alta frequência em cabos subterrâneos de alta tensão. A análise se desenvolve no domínio da frequência e a conversão ao domínio do tempo se realiza utilizando a transformada rápida de Fourier. $\mathrm{O}$ cabo se modela como uma linha de transmissão multicondutora, que utiliza a análise modal e a teoria de redes dos portos. As formulações aproximadas de impedância interna que se analisam são as propostas por Gary e Wedepohl-Wilcox, as quais são utilizadas no modelado de linhas de transmissão aéreas e subterrâneas, respectivamente. Para efetuar a análise, realiza-se uma comparação das formulações aproximadas com o modelo exato de impedância interna e determina-se o erro relativo de cada formulação tanto para os valores reais quanto para os imaginários. Os resultados mostram que a formulação aproximada proposta por Gary para o modelado de linhas de transmissão aéreas pode-se utilizar em cabos subterrâneos para analisar fenômenos transitórios eletromagnéticos de alta frequência com resultados aceitáveis. Para realizar o estudo comparativo das fórmulas aproximadas de impedância interna, propõe-se uma série de estudos de caso nos quais se determina a magnitude da voltagem transitória, o tempo de viagem e a atenuação da onda em linhas monofásicas subterrâneas que utilizam diferentes longitudes.

Palavras-chave: cabos subterrâneos, erro relativo, formulações aproximadas, impedância interna, transitórios de alta frequência. 


\section{Introducción}

Los fenómenos transitorios que se presentan en los sistemas de transmisión subterráneos se deben básicamente a maniobras de interruptores y fallas de aislamiento. Para evaluar estas tensiones y corrientes transitorias se requiere modelar el cable de manera similar al caso de líneas aéreas. Los sistemas subterráneos de transmisión tienen cierta similitud con los sistemas de tierra, ya que ambos se encuentran enterrados a cierta profundidad del nivel del suelo y sus campos electromagnéticos se ven afectados por la resistividad y permitividad del medio. Uno de los parámetros más importantes en el modelado de los cables de alta tensión es la impedancia de retorno por tierra, la cual se origina por las corrientes circulantes en la misma [1]. El cálculo de la impedancia de retorno por tierra fue tratado por Pollaczek [2], quien propone como solución una integral que no admite una solución analítica cerrada. Sin embargo, en la literatura existen varios autores que proponen diferentes soluciones aproximadas para calcular este parámetro por medio de diversas fórmulas recursivas.

Los primeros reportes de estudios del comportamiento transitorio de conductores enterrados fueron presentados en la década de los treinta, los cuales fueron realizados principalmente por Bewley [3], Bellaschi [4], Rudenberg [5] y Sunde [6]. Bewley [3] realizó pruebas experimentales que proporcionaron información importante acerca del comportamiento transitorio de los conductores enterrados. Estos estudios se centraron en estimar la impedancia de conductores enterrados mediante la derivación de una expresión para su cálculo. Años después, Bellaschi [4] obtuvo la respuesta transitoria de un conductor enterrado mediante una función escalón como fuente de excitación.

$\mathrm{El}$ análisis del comportamiento transitorio de sistemas de transmisión subterráneos no se encuentra tan extendido como en el caso de los sistemas aéreos. Básicamente existen dos técnicas: la primera, propuesta por Wedepohl y Wilcox [7], y la segunda, por Marti [8]. Estas se basan en un análisis en el dominio de la frecuencia. Para el análisis transitorio es necesario definir la matriz de impedancia serie $\mathrm{Z}$ del sistema de transmisión subterráneo, que está constituida por varias submatrices, en una de las cuales se considera la impedancia interna del conductor central del cable subterráneo. La impedancia interna de conductores cilíndricos para grandes parámetros puede ser calculada mediante ciertos modelos aproximados, los cuales no involucran el cálculo de las funciones de Bessel, funciones de
Bessel modificadas o funciones de Kelvin y por lo tanto permiten determinar la impedancia interna de una manera más sencilla con poco esfuerzo computacional. Sin embargo, estos modelos no son exactos y presentan cierto margen de error, por lo que son clasificados como modelos de baja precisión, tales como los propuestos por Nahman y Holt [9], Semlyen y Deri [10], Wedepohl y Wilcox [11]; y modelos de alta precisión, como los propuestos por Vujević, Boras y Sarajčev [12], Mingli y Yu [13], Knight [14].

Torres, Jardines y Guardado [15] utilizan una metodología para el estudio de la propagación de pulsos electromagnéticos en cables subterráneos basada en teoría de líneas de transmisión multiconductoras, análisis modal, redes de dos puertos y la transformada rápida de Fourier, la cual será utilizada en el presente artículo para realizar el estudio comparativo de las formulaciones aproximadas de impedancia interna de cables subterráneos, a fin de evaluar su precisión en estudios de transitorios electromagnéticos ultrarrápidos.

\section{Materiales y métodos}

\subsection{Características físicas de los cables de alta tensión}

Por lo general, un sistema de transmisión de energía eléctrica subterráneo consta de tres cables coaxiales concéntricos, enterrados a la misma profundidad, tal como se muestra en la figura 1.

Cada cable está compuesto por dos conductores metálicos concéntricos en el cual uno es el conductor central y el otro la pantalla conductora o armadura, separados por aislantes. En la figura 2 se muestra la configuración geométrica de los cables coaxiales del sistema de la figura 1.

Los aislantes utilizados en los cables de alta tensión se caracterizan por presentar bajas pérdidas dieléctricas. Entre los materiales aislantes más comúnmente utilizados se encuentra el cloruro de polivinilo (PVC), el polietileno ( $\mathrm{PE}$ ) y el polietileno de cadena cruzada (XLPE). En la tabla 1 se presentan la permitividad relativa de estos aislantes a $20^{\circ} \mathrm{C}$ y el factor de pérdidas típico a una frecuencia de $50 \mathrm{~Hz}$ Marti [8].

A partir de estas características físicas y eléctricas, se determina la impedancia serie $Z$ y la admitancia paralelo $Y$ del cable de alta tensión, haciendo las siguientes consideraciones: 
- Las pérdidas en los dieléctricos involucrados son despreciables.

- El conductor tiene una permeabilidad constante.
- Los cables son longitudinalmente uniformes, paralelos entre sí y a la superficie de la tierra.

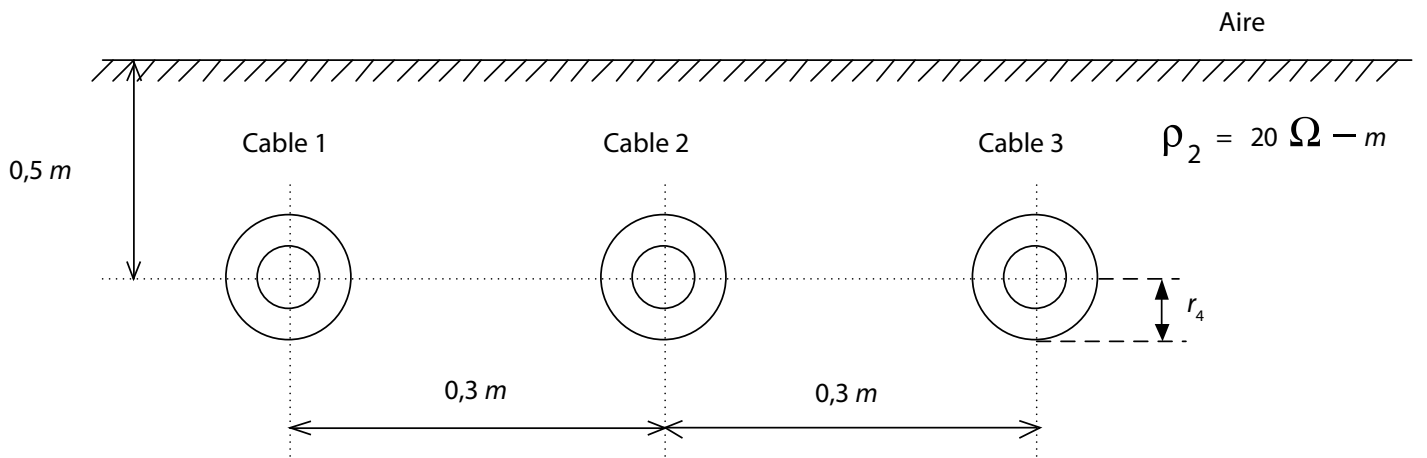

Figura 1. Sistema de transmisión subterráneo

Fuente: los autores

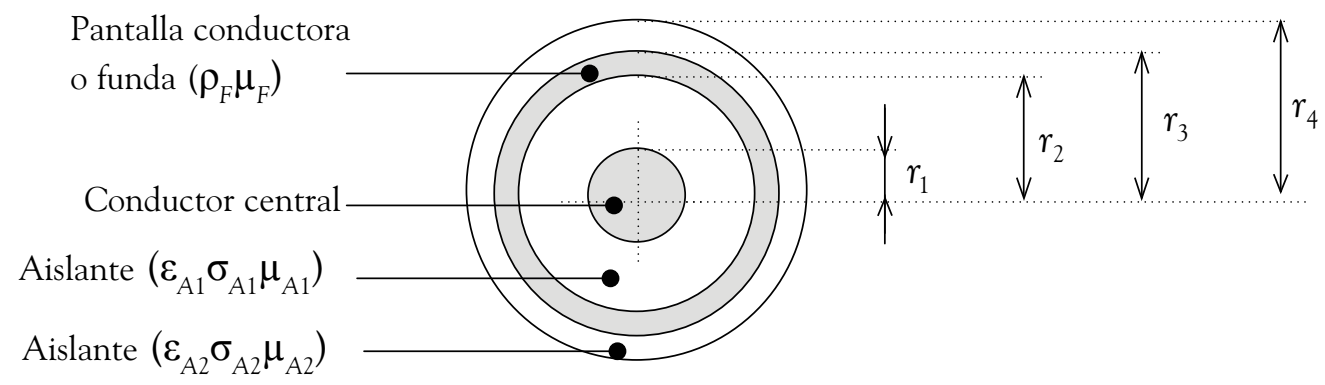

Figura 2. Sección transversal de un cable coaxial de alta tensión

Fuente: los autores

Tabla 1. Características físicas de aislantes utilizados en cables de alta tensión

\begin{tabular}{|l|c|c|}
\hline \multicolumn{1}{|c|}{ Material aislante } & Permitividad relativa $\left(\mathbf{2 0}^{\circ} \mathbf{C}\right)$ & $\begin{array}{c}\text { Factor de pérdida tan } \boldsymbol{~} \\
\left(\mathbf{5 0} \mathbf{~ H z}-\mathbf{2 0} \mathbf{~}^{\circ} \mathbf{C}\right)\end{array}$ \\
\hline Goma de butilo & $3,0-4,0$ & 0,05 \\
Aceite aislante & $2,2-2,8$ & $0,001-0,002$ \\
Papel impregnado en aceite aislante & $3,3-4,2$ & $0,003-0,008$ \\
Cloruro de polivinilo (Pvc) & $3,0-4,0$ & $0,002-0,10$ \\
Polietileno & 2,3 & 0,0002 \\
Polietileno de cadena cruzada & 2,4 & 0,0004 \\
\hline
\end{tabular}

Fuente: Marti [8] 


\subsection{Impedancia serie de cables de alta tensión}

La impedancia serie $Z$ del sistema de transmisión se expresa a una frecuencia angular dada y está constituida por $N^{2}$ número de submatrices. En la construcción de las submatrices de la diagonal principal se requiere determinar primeramente siete magnitudes de impedancias, las cuales resultan de la interacción del campo eléctrico y magnético entre el núcleo, la pantalla conductora y el aislamiento del cable. Estos términos son los siguientes [11]:

$\mathrm{z}_{1} \equiv$ Impedancia interna del conductor central.

$\mathrm{z}_{2} \equiv$ Impedancia debida al flujo en el aislamiento interior debido a la variación del campo magnético con el tiempo.

$\mathrm{z}_{3} \equiv$ Impedancia interna de la funda interior.

$\mathrm{z}_{4} \equiv$ Impedancia mutua de la pantalla conductora. $\mathrm{z}_{5} \equiv$ Impedancia interna de la funda exterior.

$\mathrm{z}_{6} \equiv$ Impedancia del aislamiento exterior debida a la variación del flujo magnético con el tiempo.

$\mathrm{z}_{\mathrm{T}} \equiv$ Impedancia debida al retorno por tierra.

La impedancia interna del conductor cilíndrico-cen$\operatorname{tral} Z_{1}$ se puede calcular mediante diversos modelos aproximados. En este trabajo se utilizan dos modelos aproximados para evaluarlos en cuanto a su precisión en el análisis transitorio de sistemas de transmisión subterráneos.

\subsection{Formulaciones clásicas de la impedancia serie}

A bajas frecuencias, $z_{1}$ es aproximadamente igual a la impedancia del conductor principal en corriente directa. Para frecuencias elevadas $z_{1}$ se obtiene por medio de la expresión (1), la cual considera el efecto piel [11]:

$$
\begin{aligned}
& z_{1}=\frac{\rho_{N} m_{1}}{2 \pi r_{1}} \frac{I_{0}\left(m_{1} r_{1}\right)}{I_{1}\left(m_{1} r_{1}\right)} \quad \Omega / m \\
& z_{2}=\frac{j \omega \mu_{A 1}}{2} \operatorname{In}\left(\frac{r_{2}}{r_{1}}\right) \quad \Omega / m
\end{aligned}
$$

donde $m_{1}$ es la constante de propagación del conductor dada por,

$$
m_{1}=\sqrt{\frac{j \omega \mu_{A 1}}{\rho_{N}}}
$$

En las expresiones anteriores $\rho_{\mathrm{N}}$ representa la resistividad del núcleo del cable, $\omega$ corresponde a la frecuencia angular, $\mu_{\mathrm{A} 1}$ es la permeabilidad del aislante 1, $r_{1}$ es el radio del núcleo y $r_{2}$ es el radio del aislante. Los términos $I_{0}$ e $I_{1}$ corresponden a la función de Bessel modificada de primera clase de orden cero y primer orden, respectivamente.

Para la impedancia de la pantalla conductora $\mathrm{z}_{3}, \mathrm{z}_{4}$ y $z_{5}$ se utilizan las formulas clásicas siguientes:

$$
\begin{gathered}
z_{3}=\frac{\frac{\rho_{F} m_{2}}{2 \pi r_{2}}\left\{I_{0}\left(m_{2} r_{2}\right) K_{1}\left(m_{2} r_{3}\right)+K_{0}\left(m_{2} r_{2}\right) I_{1}\left(m_{2} r_{3}\right)\right\}}{D} \Omega / m \\
z_{4}=\frac{\left(\frac{\rho_{F}}{2 \pi r_{2} r_{3}}\right)}{D} \Omega / \mathrm{m} \\
z_{5}=\frac{\frac{\rho_{F} m_{2}}{2 \pi r_{3}}\left\{I_{0}\left(m_{2} r_{3}\right) K_{1}\left(m_{2} r_{2}\right)+K_{0}\left(m_{2} r_{3}\right) I_{1}\left(m_{2} r_{2}\right)\right\}}{D} \Omega / m
\end{gathered}
$$




$$
\begin{gathered}
z_{6}=\frac{j \omega \mu_{A 2}}{2} \operatorname{In}\left(\frac{r_{4}}{r_{3}}\right) \quad \Omega / m \\
D=I_{1}\left(m_{2} r_{3}\right) K_{1}\left(m_{2} r_{2}\right)-I_{1}\left(m_{2} r_{2}\right) K_{1}\left(m_{2} r_{3}\right)
\end{gathered}
$$

donde $m_{2}$ es la constante de propagación de la pantalla conductora dada por,

$$
m_{2}=\sqrt{\frac{j \omega \mu_{A 2}}{\rho_{F}}}
$$

En las anteriores expresiones $\rho_{\mathrm{F}}$ representa la resistividad del núcleo de la funda, $\mu_{\mathrm{A} 2} \mathrm{y}_{4}$ corresponden, en forma respectiva, a la permeabilidad y el radio del aislante 2, mientras que $\mathrm{r}_{3}$ es el radio de la funda. $K_{0} \mathrm{y}$ $K_{1}$ son las funciones de Bessel modificadas de segunda clase de orden cero y uno, respectivamente.

\subsection{Formulaciones aproximadas de impedancia interna}

Existen diversas formulaciones aproximadas para el cálculo de la impedancia interna de conductores cilíndricos, los cuales permiten determinar dicha impedancia de una manera fácil, ya que no consideran a las funciones de Bessel o Bessel modificadas. Un modelo aproximado para la impedancia interna de conductores de una línea de transmisión aérea monofásica es dado por la ecuación (10). Este modelo es una aproximación simple y presenta un error considerable en el cálculo de la impedancia interna Johnson y Gram [16].

$$
Z_{c} \approx Z_{c d}+Z_{c a} \approx \frac{\rho_{N}}{\pi r_{1}^{2}}+\frac{\rho_{N} m_{1}}{2 \pi r_{1}} \Omega / m
$$

Una mejor aproximación de la anterior ecuación, dada por (11), es proporcionada en una formulación descrita por Gary [17], la cual es utilizada para el cál- culo de los parámetros eléctricos de una línea de transmisión aérea monofásica con conductores de sección transversal circular o cilíndricos.

$$
Z_{C 1} \approx \sqrt{Z_{c d}^{2}+Z_{c a}^{2}} \approx \sqrt{\left(\frac{\rho_{N}}{\pi r_{1}^{2}}\right)^{2}+\left(\frac{\rho_{N} m_{1}}{2 \pi r_{1}}\right)^{2}} \Omega / m
$$

donde $r_{1}$ corresponde al radio del conductor desnudo de la línea de transmisión aérea, que es equivalente al radio del conductor central del cable subterráneo mostrado en la figura 2. Los términos $\rho_{N} \mathrm{y} m_{1}$ son, respectivamente, la resistividad del material del conductor y la constante de propagación del conductor dada por la expresión (3). Es importante mencionar que de acuerdo con la ecuación anterior, la impedancia inter- na $Z_{C l}$ se calcula considerando la impedancia de corriente directa $Z_{c d}$ y la impedancia de corriente alterna $Z_{c a}$ las cuales también pueden ser referidas como impedancia de baja y alta frecuencia, respectivamente.

Wedepohl y Wilcox [11] propusieron otro modelo aproximado de impedancia interna con el cual se obtienen valores aceptables para el análisis de líneas de transmisión subterráneas. 


$$
z_{C 2}=\frac{\rho_{N} m_{1}}{2 \pi r_{1}} \operatorname{coth}\left(0,777 m_{1} r_{1}\right)+\frac{0,356 \rho_{N}}{\pi r_{1}^{2}} \Omega / m
$$

El primer término de este modelo aproximado exhibe propiedades similares a las expresiones de impedancia interna dadas por (1) y (11), ya que en altas frecuencias este término tiende a la impedancia $Z_{c a}$, que es conocida como efecto piel, mientras que para bajas frecuencias representa la impedancia $Z_{c d}$. El segundo término, el cual es constante y no depende de la frecuencia, mejora la precisión del modelo aproximado porque corrige la impedancia de corriente directa.

Se debe notar que el modelo aproximado dado por (11) es comúnmente utilizado en el análisis de líneas de transmisión aéreas, mientras que el modelo dado por la ecuación (12) es útil en el análisis de cables subterráneos; sin embargo, debido a la similitud en el comportamiento de ambos modelos aproximados, surge la necesidad de llevar a cabo una evaluación de los dos modelos presentados en (11) y (12) para el análi- sis transitorio de líneas de transmisión subterráneas, lo cual finalmente permite determinar si el modelo aproximado utilizado en las líneas de transmisión aérea es confiable para el análisis de transitorios electromagnéticos de cables subterráneos.

\subsection{Impedancia de retorno por tierra}

La impedancia de retorno por tierra se origina por las corrientes circulantes en el plano de tierra de la figura 3. Para evaluar estas corrientes, Pollaczek [2] desarrolló una integral que lleva su nombre. Sin embargo, esta integral no acepta una solución analítica cerrada.

Considerando el sistema en la figura 3, se obtienen las expresiones (13) a (17) para la impedancia propia y mutua, así como para la densidad de corriente en el suelo (Wedepohl y Wilcox [11], Uribe [18]).

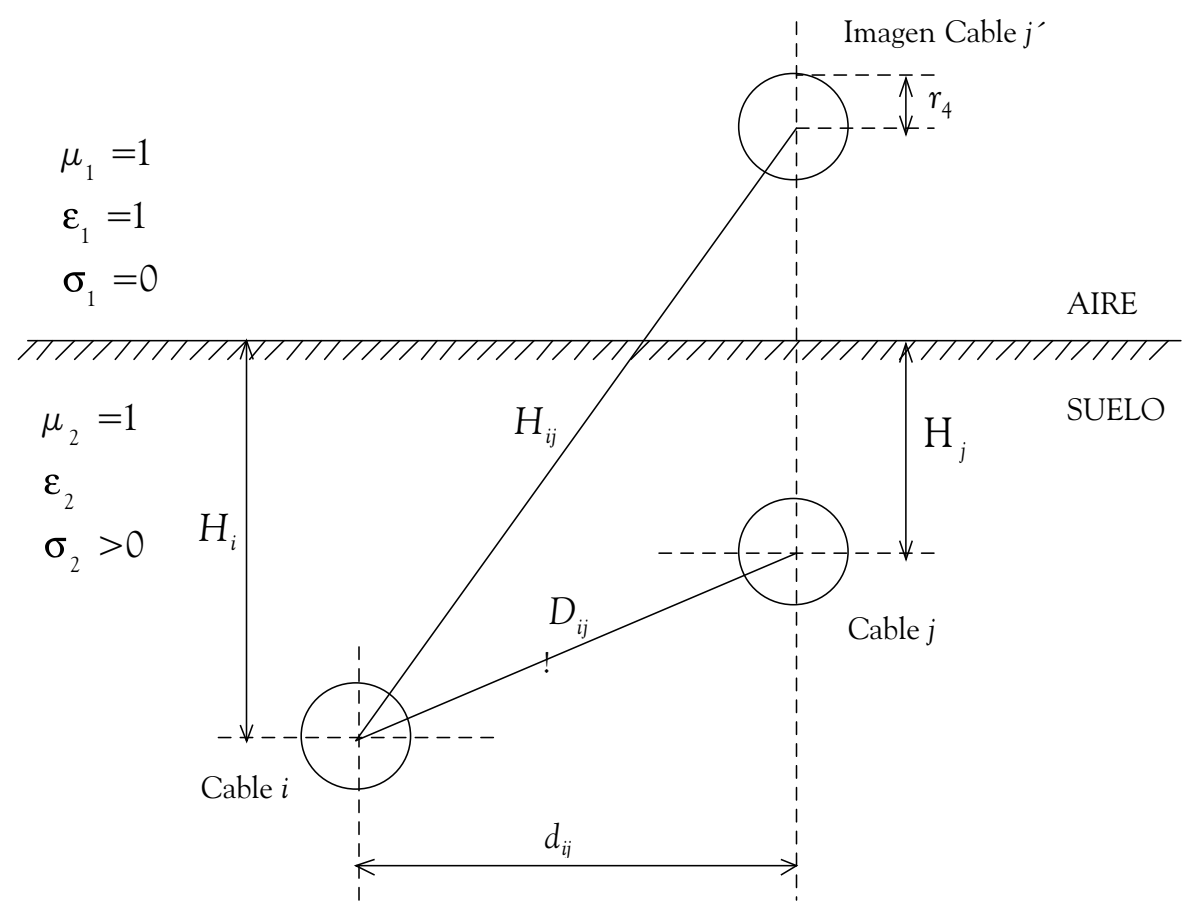

Figura 3. Sistema de transmisión subterráneo compuesto por dos cables

Fuente: Pollaczek [2] 


$$
\begin{aligned}
& Z t_{\text {PROPIA }}=\frac{j \omega \mu_{0}}{2 \pi}\left[K_{0}\left(\frac{r_{4}}{p}\right)-K_{0}\left(\frac{2 H_{i}}{p}\right)+J_{\text {PROPIA }}\right] \Omega / m \\
& J_{\text {PROPIA }}=\int_{-\infty}^{\infty} \frac{\exp \left|-2 H_{i} \sqrt{\beta^{2}+\frac{1}{p^{2}}}\right|}{|\beta|+\sqrt{\beta^{2}+\frac{1}{p^{2}}}} \cdot \exp \left(j \beta r_{4}\right) d \beta \\
& p=\frac{1}{\sqrt{j \omega \mu \sigma_{\text {SUELO }}}}
\end{aligned}
$$

En las anteriores ecuaciones $H_{i}$ es la profundidad a es el radio del aislante 2, la conductividad del suelo es la que está enterrado el cable $i$, los términos $\mu_{0} \mathrm{y} \mu$ repre- $\quad$ representada por $\sigma_{\text {SUELO }}$ y $K_{0}$ es la función modificada sentan la permeabilidad del vacío y del suelo, respecti- de Bessel de segunda clase y orden cero y vamente. $\beta$ corresponde a la variable de integración, $r_{4}$

$$
\begin{aligned}
Z t_{\text {MUTUA }} & =\frac{j \omega \mu_{0}}{2 \pi}\left[K_{0}\left(\frac{D_{i j}}{p}\right)-K_{0}\left(\frac{H_{i j}}{p}\right)+J_{\text {MUTUA }}\right] \Omega / m \\
J_{\text {MUTUA }} & =\int_{-\infty}^{\infty} \frac{\exp \mid-\left(H_{i}+H_{j}\right) \sqrt{\beta^{2}+\frac{1}{p^{2}} \mid}}{|\beta|+\sqrt{\beta^{2}+\frac{1}{p^{2}}}} \cdot \exp (j \beta x) d \beta
\end{aligned}
$$

donde $H_{\mathrm{ij}}$ es la distancia del cable a su imagen y $D_{\mathrm{ij}}$ corresponde a la distancia entre cables $i$ y $j$.

En la literatura especializada se encuentran diversas aproximaciones para la evaluación de la impedancia de retorno por tierra. El modelo propuesto por Saad, Gaba y Giroux [19] es considerado aceptable para cables subterráneos, debido a la buena aproximación en sus resultados con respecto a la integral de Pollaczek. Por tal razón, en este artículo se utilizará este modelo para calcular la impedancia de retorno por tierra, el cual es dado por las ecuaciones (18) y (19).

$$
\begin{aligned}
& Z_{T_{\text {PROPA }}}=\frac{j \omega \mu_{0}}{2 \pi}\left[K_{0}\left(r_{4} m\right)+\frac{2}{4+\left(r_{4} m\right)^{2}} \cdot \exp \left(-2 H_{i} m\right)\right] \Omega / m \\
& Z_{T_{\text {MUTUA }}}=\frac{j \omega \mu_{0}}{2 \pi}\left[K_{0}\left(D_{i j} m\right)+\frac{2}{4+\left(d_{i j} m\right)^{2}} \cdot \exp \left(-2 h_{i j} m\right)\right] \Omega / m
\end{aligned}
$$

donde $h_{\mathrm{ij}}$ es la profundidad promedio para el caso de las impedancias mutuas y ds es la distancia horizontal entre cables $i \mathrm{y} j$ 


\subsection{Construcción de la matriz de impedancia} serie

En la construcción de la matriz de impedancia serie se considera el circuito equivalente de un cable coaxial mostrado en la figura 4 [11].

A partir de esta figura se construyen las siguientes submatrices que corresponden a la impedancia propia.

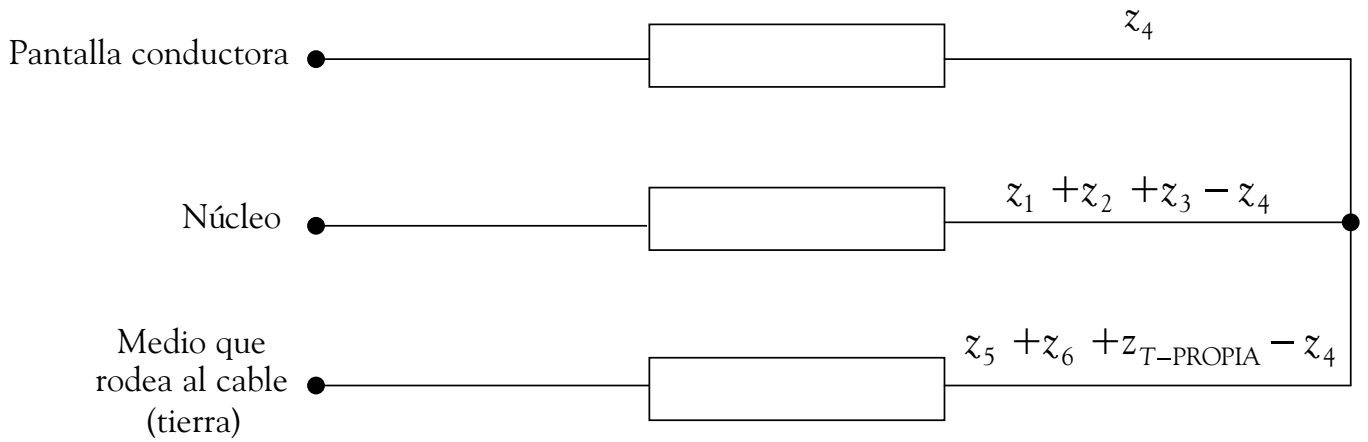

Figura 4. Circuito equivalente de un cable coaxial

Fuente: Wedepohl y Wilcox [11]

$$
Z_{i i}=\left[\begin{array}{cc}
z_{1}+z_{2}+z_{3}+z_{5}+z_{6}+z_{7}-2 z_{4} & z_{5}+z_{6}+z_{7}-z_{4} \\
z_{5}+z_{6}+z_{7}-z_{4} & z_{5}+z_{6}+z_{T-P R O P I A}
\end{array}\right]
$$

Para la impedancia mutua se tiene,

$$
Z_{T-M U T U A i j}=\left[\begin{array}{cc}
z_{T-M U T U A i j} & z_{T-M U T U A i j} \\
z_{T-M U T U A i j} & z_{T-M U T U A i j}
\end{array}\right]
$$

La impedancia serie se construye ensamblando a lo largo de la diagonal principal las submatrices de la impedancia propia $Z_{\text {ii }}$ y fuera de la diagonal las subma- trices de la impedancia mutua $Z_{\mathrm{ij}}$, las cuales corresponden a los acoplamientos entre los cables.

$$
Z=\left[\begin{array}{cccc}
Z_{11} & Z_{i j} & Z_{i j} & \cdot \\
Z_{i j} & Z_{22} & Z_{i j} & \cdot \\
Z_{i j} & Z_{i j} & Z_{33} & \cdot \\
\cdot & \cdot & \cdot & Z n n
\end{array}\right]
$$

\subsection{Construcción de la matriz de admitancia paralelo}

La dimensión de las submatrices de la admitancia paralelo son de 2x2, ya que están en función del número de aislantes en el cable. 


$$
Y_{i i}=\left[\begin{array}{cc}
y_{1} & -y_{1} \\
-y_{1} & y_{1}+y_{2}
\end{array}\right]
$$

De manera general, las submatrices son de la siguiente forma,

$$
Y_{i i}=\left[\begin{array}{cccc}
y_{1} & -y_{1} & 0 & 0 \\
-y_{1} & y_{1}+y_{2} & -y_{2} & 0 \\
0 & -y_{2} & y_{2}+y_{3} & -y_{N} \\
0 & 0 & -y_{N} & y_{N}+y_{N+1}
\end{array}\right]
$$

donde $\mathrm{N}$ es el número de aislante del cable, mientras que los elementos de la matriz de admitancia paralelo son dados por las siguientes expresiones,

$$
\begin{gathered}
y_{i}=g_{i}+j \omega C_{i} \quad(i=1,2) \\
C_{i}=\frac{2 \pi \varepsilon_{i}}{\operatorname{In}\left(\frac{r_{2 i}}{r_{(2 i-1)}}\right)} \\
g_{i}=\omega C \cdot \tan (\delta)
\end{gathered}
$$

Debe destacarse que los términos $\mathrm{r}_{2 \mathrm{i}}, \mathrm{r}_{(2 \mathrm{i}-1)}, \varepsilon_{\mathrm{Ai}}, \sigma_{\mathrm{Ai}}$ se obtienen de la figura 2 , donde $\delta$ es el factor de pérdidas del aislamiento.

De acuerdo con el número de cables existentes en el sistema de transmisión, la matriz de admitancia pa- ralelo se construye a partir de las admitancias propias del cable, despreciando los acoplamientos mutuos entre los mismos, por lo que esta matriz adopta la forma siguiente,

$$
Y=\left[\begin{array}{cccc}
Y_{11} & 0 & 0 & \cdot \\
0 & Y_{22} & 0 & \cdot \\
0 & 0 & Y_{33} & \cdot \\
\cdot & \cdot & \cdot & Y n n
\end{array}\right]
$$

donde $n$ es el número de cables en el sistema.

\section{Resultados}

\subsection{Análisis de las formulaciones aproximadas de impedancia interna}

El análisis de las formulaciones o modelos aproximados de impedancia interna, dados por las ecuaciones (10) y (11), es realizado comparando los valores de impedancia obtenidos por dichos modelos y los determinados por el modelo exacto dado por (1). Además, como parte del análisis comparativo se determina el error relativo que presentan las formulaciones aproximadas en función de la frecuencia. Para llevar a cabo la comparación y determinar el error relativo, se consideró la permeabilidad del espacio libre y se emplearon los datos referentes a las características eléctricas y dimensionales del conductor acsr $636-\mathrm{mcm}$, 
conocido también como Grosbeak, el cual tiene una resistividad de $0,0885 \Omega / \mathrm{km}$ y un radio de $10,921 \mathrm{~mm}$ [20].

La figura 5 muestra una comparación gráfica entre los valores reales e imaginarios de la impedancia interna obtenida por medio de los modelos aproximados y del modelo exacto en función de la frecuencia. El rango de frecuencia en el que fue evaluada la impedancia interna de los modelos fue de 0 a $100 \mathrm{MHz}$. En la figura se observa que los dos modelos aproximados de impedancia interna presentan un comportamiento muy similar al modelo exacto, tanto en los valores reales como en los va- lores imaginarios, los cuales corresponden a la resistencia y a la inductancia, respectivamente. En el acercamiento de la figura $5 \mathrm{a}$ y $5 \mathrm{~b}$ se observa que ambas formulaciones aproximadas tienen un mejor comportamiento con los valores reales, mientras que con los valores imaginarios presenta una diferencia mayor. En general, se puede decir que las dos formulaciones aproximadas tienen buen comportamiento en todo el espectro de frecuencias considerado; sin embargo, la mayor discrepancia de ambos modelos con el modelo exacto es presentada en el rango de frecuencia aproximado de 0,2 a 1,96 MHz.

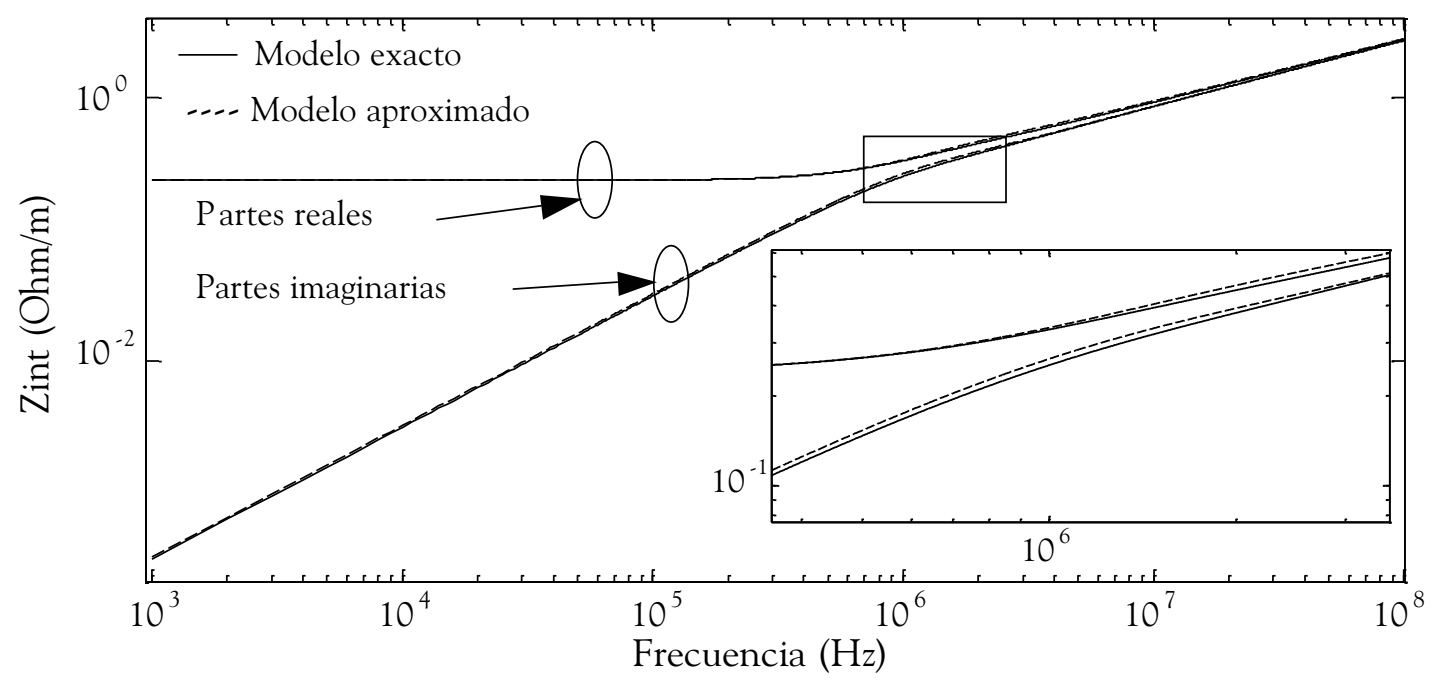

a) Impedancia interna de la formulación exacta y aproximada, ecuaciones (1) y (11)

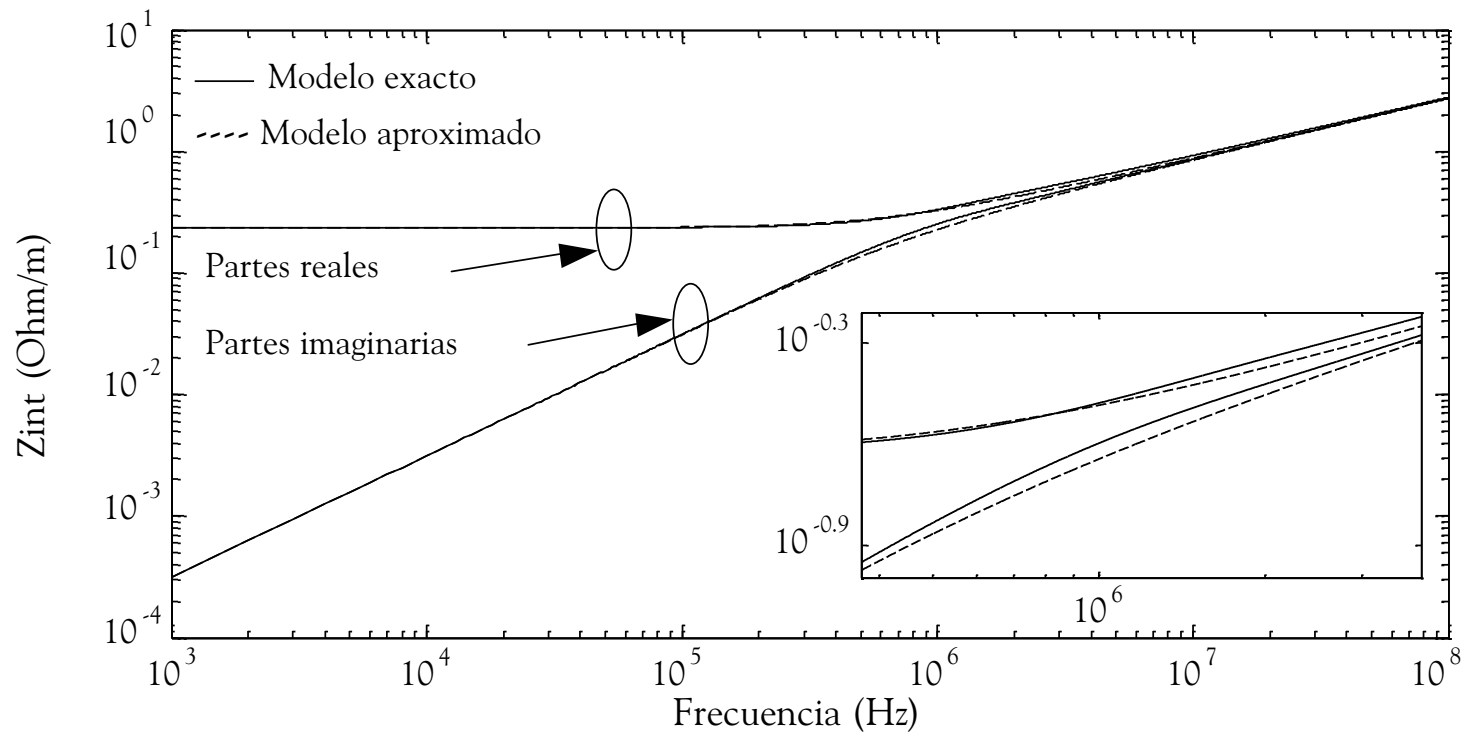

b) Impedancia interna de la formulación exacta y aproximada, ecuaciones (1) y (12)

Figura 5. Impedancia interna en función de la frecuencia obtenida por medio de las formulaciones aproximadas y el modelo exacto Fuente: los autores 
El error relativo del modelo de impedancia interna es determinado de acuerdo con la ecuación (29), donde dicho error es obtenido de la siguiente manera,

$$
\text { Error }=\frac{\left|Z_{e}-Z_{a}\right|}{Z_{e}} X 100 \%
$$

En esta ecuación los términos $\mathrm{Z}_{\mathrm{e}}$ y $\mathrm{Z}_{\mathrm{a}}$ corresponden a la impedancia obtenida por medio de las formulaciones aproximadas y el modelo exacto, respectivamente. Estos valores son dependientes de la frecuencia, por lo que el error relativo dado por (29) también es una función de esta variable. La figura 6 presenta dos gráficas del error relativo para valores reales y valores imaginarios de las formulaciones aproximadas de impedancia interna.

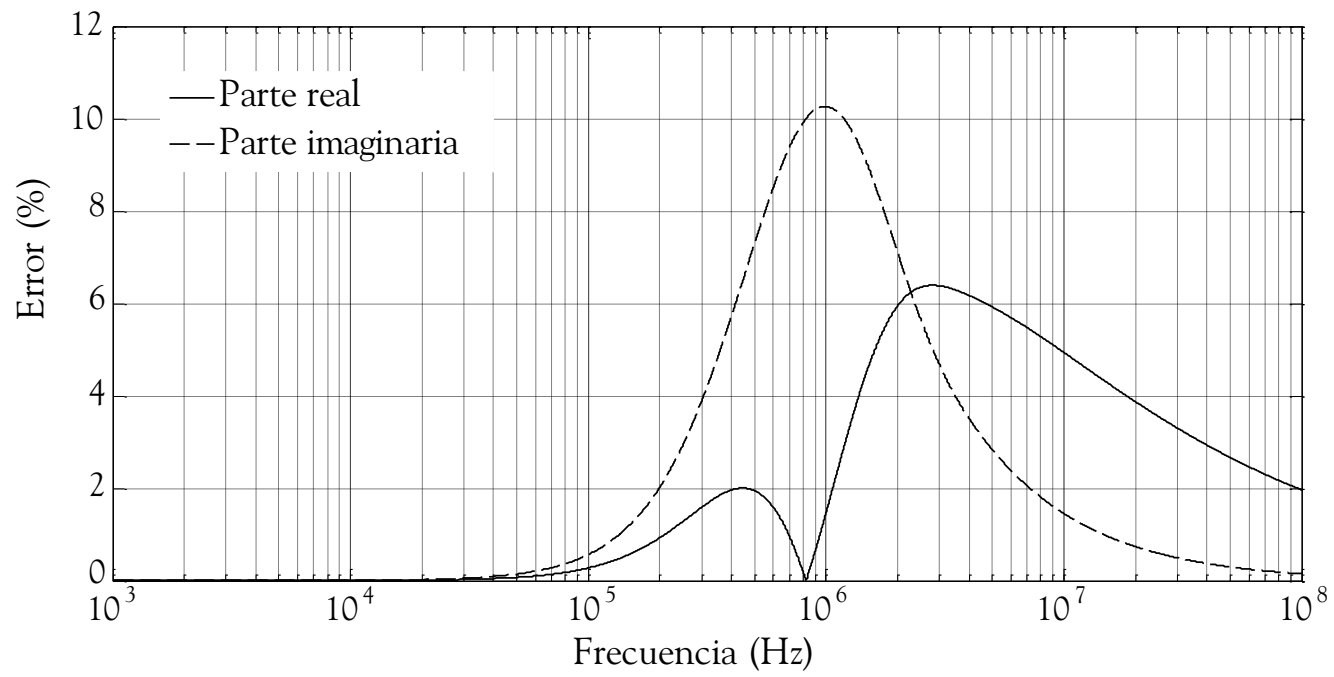

Error relativo de la formulación aproximada dada por la ecuación (11)

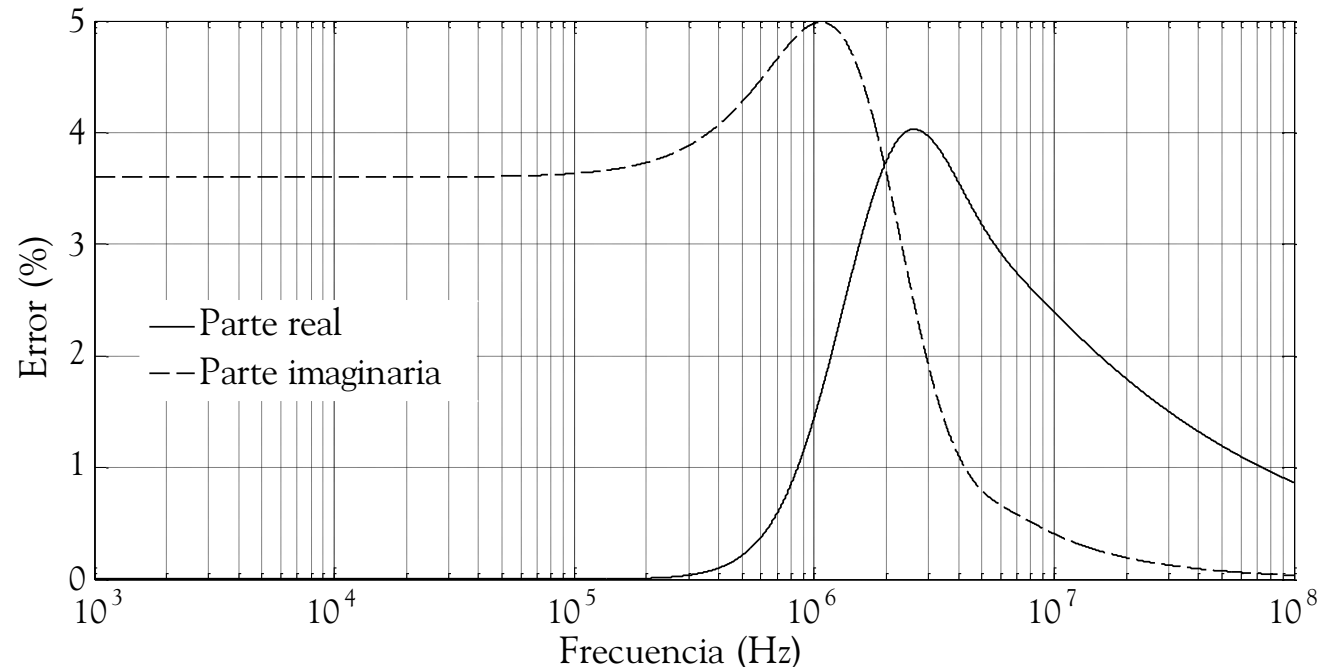

Error relativo de la formulación aproximada dada por la Ecuación (12)

Figura 6. Error relativo de las formulaciones aproximadas de impedancia interna Fuente: elaboración propia

Las curvas de error de la figura 6 muestran que en ambas formulaciones aproximadas el error relativo referente a los valores imaginarios es mayor que el error relativo correspondiente a los valores reales de impedancia interna, por lo que se puede generalizar que tales formulaciones tienen un mejor comportamiento con los valores reales. Sin embargo, es necesario notar que ambas formulaciones aproximadas de impedancia interna tienen un 
error relativo menor para valores reales en bajas frecuencias y para valores imaginarios en altas frecuencias, lo cual se traduce en que dichas formulaciones aproximadas tienen mejor comportamiento para los valores reales en bajas frecuencias, mientras que en altas frecuencias presenta un mejor comportamiento para los valores imaginarios.

Para el caso de la formulación descrita por Gary [17] y dada por la ecuación (11), figura 6a, en bajas frecuencias el error relativo del modelo aproximado es muy bajo tanto para valores reales como para valores imaginarios, pero en aproximadamente $100 \mathrm{kHz}$ el error comienza a crecer para los valores reales y para los valores imaginarios. Sin embargo, en $824 \mathrm{kHz}$ el error relativo de los valores reales es cero y el error de los valores imaginarios es prácticamente el error relativo máximo. En el caso de los valores reales, el error relativo comienza a crecer después de $824 \mathrm{kHz}$ hasta llegar a su error máximo en aproximadamente 2,82 $\mathrm{MHz}$ para posteriormente disminuir con el aumento de la frecuencia. En el caso de los valores imaginarios, el error relativo máximo se encuentra en un valor de frecuencia aproximado de $1 \mathrm{MHz}$ y después de este valor el error comienza a disminuir con el incremento de la frecuencia.

En lo que se refiere a la formulación aproximada de la impedancia interna propuesta por Wedepohl y Wilcox [11], ecuación (12), la figura $6 \mathrm{~b}$ muestra que para los valores imaginarios existe un error relativo de $3,6 \%$ desde $1 \mathrm{kHz}$ hasta aproximadamente 100 $\mathrm{kHz}$, mientras que en el mismo rango de frecuencias el error relativo referido a los valores reales es muy pequeño, menor a $1 \times 10-3 \%$. Con el aumento de la frecuencia el error relativo comienza a crecer alcanzando el valor máximo para valores imaginarios y reales en aproximadamente $1,07 \mathrm{MHz}$ y $2,62 \mathrm{MHz}$, respectivamente.

Es importante mencionar que los errores relativos máximos de la formulación aproximada descrita por Gary [17], dada por (11), son de 6,3941\% y de $10,2671 \%$ para los valores reales e imaginarios de impedancia interna, respectivamente. Mientras que para la formulación aproximada propuesta por Wedepohl y Wilcox [11], ecuación (12), el error relativo para valores reales es $4,0302 \%$ y $4,9884 \%$ para los valores imaginarios. De acuerdo con esto, es sencillo inferir que esta última formulación tiene un menor error tanto en valores reales como en valores imaginarios; sin embargo, es necesario implementar ambos modelos en un algoritmo basado en la metodología descrita en las secciones anteriores con el fin de investigar si tal diferencia de error relativo tiene un impacto importante en el análisis transitorio de cables subterráneos de alta tensión.

\subsection{Comportamiento transitorio de un cable de alta tensión}

Algunos casos de estudio son presentados con el fin de ilustrar la aplicación de las formulaciones aproximadas de impedancia interna en el análisis de transitorios de alta frecuencia de cables subterráneos de alta tensión. Se consideran tres líneas monofásicas subterráneas con longitudes de $10 \mathrm{~km}, 40 \mathrm{~km}$ y $80 \mathrm{~km}$ a una profundidad de $0,79 \mathrm{~m}$ con una resistividad del suelo de $20 \Omega-\mathrm{m}$. En todos los casos las líneas son excitadas con una función escalón de 1 pu. Los datos de la sección transversal del cable son dados en Uribe, Naredo, Moreno y Guardado [21].

Los materiales utilizados en el cable son: cobre para el conductor central, plomo para la pantalla conductora y cloruro de polivinilo (PVC) para el aislante. El modelado del sistema de trasmisión se realiza de acuerdo con Torres [1], Wedepohl y Wilcox [11] y Uribe, Naredo, Moreno y Guardado [22].

Las formulaciones aproximadas de impedancia interna dadas por las ecuaciones (11) y (12) son utilizadas en las figuras 7 y 8 , respectivamente, en las cuales se muestran los voltajes transitorios al final de la línea, el tiempo de viaje y la atenuación de la onda de voltaje.

En las figuras anteriores se observa que mientras mayor sea la longitud de la línea de transmisión subterránea, menor es la magnitud del voltaje transitorio; además, el aumento en la longitud de la línea provoca que se incremente el tiempo de desplazamiento de la onda de viaje. Por otra parte, se debe notar que en los casos de $40 \mathrm{~km}$ y $80 \mathrm{~km}$, aumenta el tiempo de la respuesta transitoria del voltaje al final de la línea.

De acuerdo con las figuras 7 y 8 y con el análisis anterior, es posible observar que en el análisis de transitorios de alta frecuencia de cables subterráneos se puede utilizar cualquiera de las dos formulaciones aproximadas de impedancia interna, ya que se obtienen resultados confiables y no se tiene una discrepancia notable en dichos resultados debido a la utilización de una u otra formulación. Es importante mencionar que la formulación aproximada de impedancia interna propuesta por Gary [17] es comúnmente utilizada en el modelado de líneas de transmisión aéreas; sin embargo, de acuerdo con los resultados de las simulaciones de los casos de estudio presentados, es posible afirmar que dicha formulación aproximada también se puede 


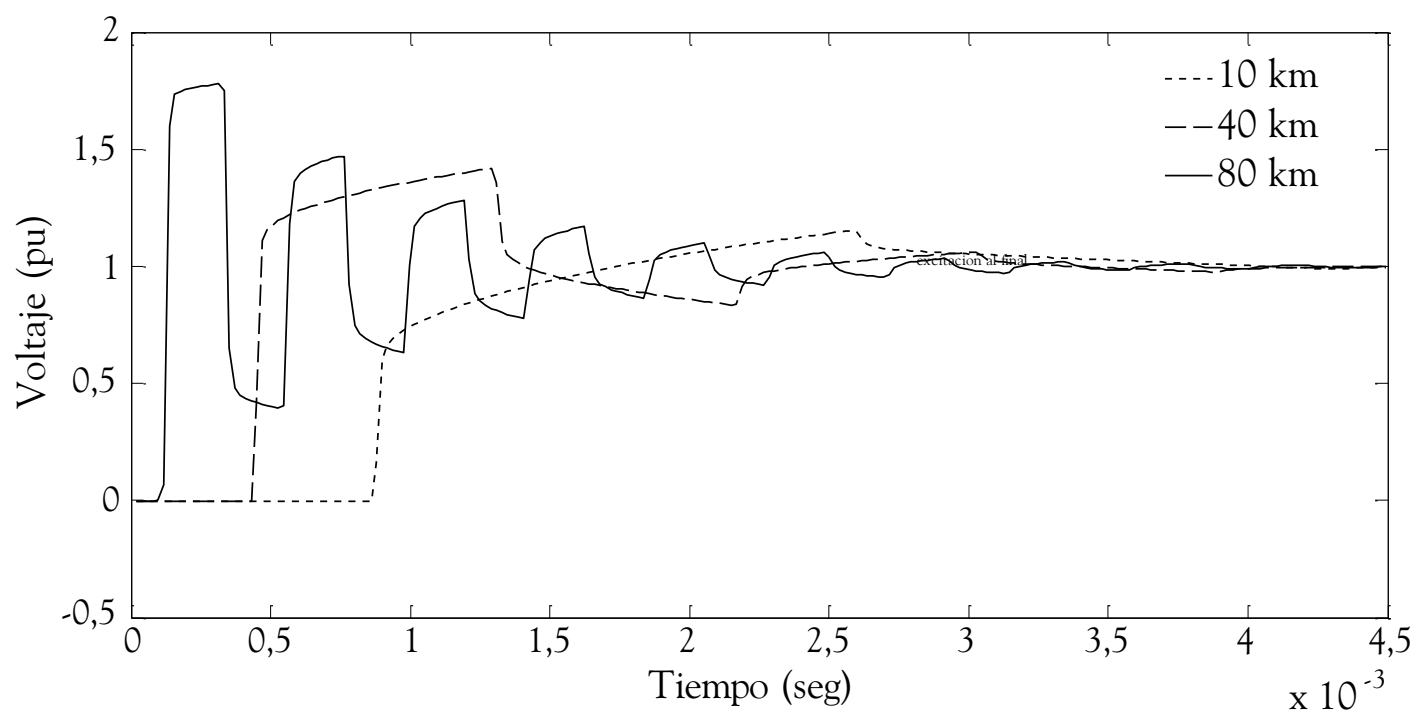

Figura 7. Respuesta transitoria del voltaje al final de la línea monofásica subterránea utilizando la formulación aproximada descrita por Gary [17], ecuación (11)

Fuente: los autores

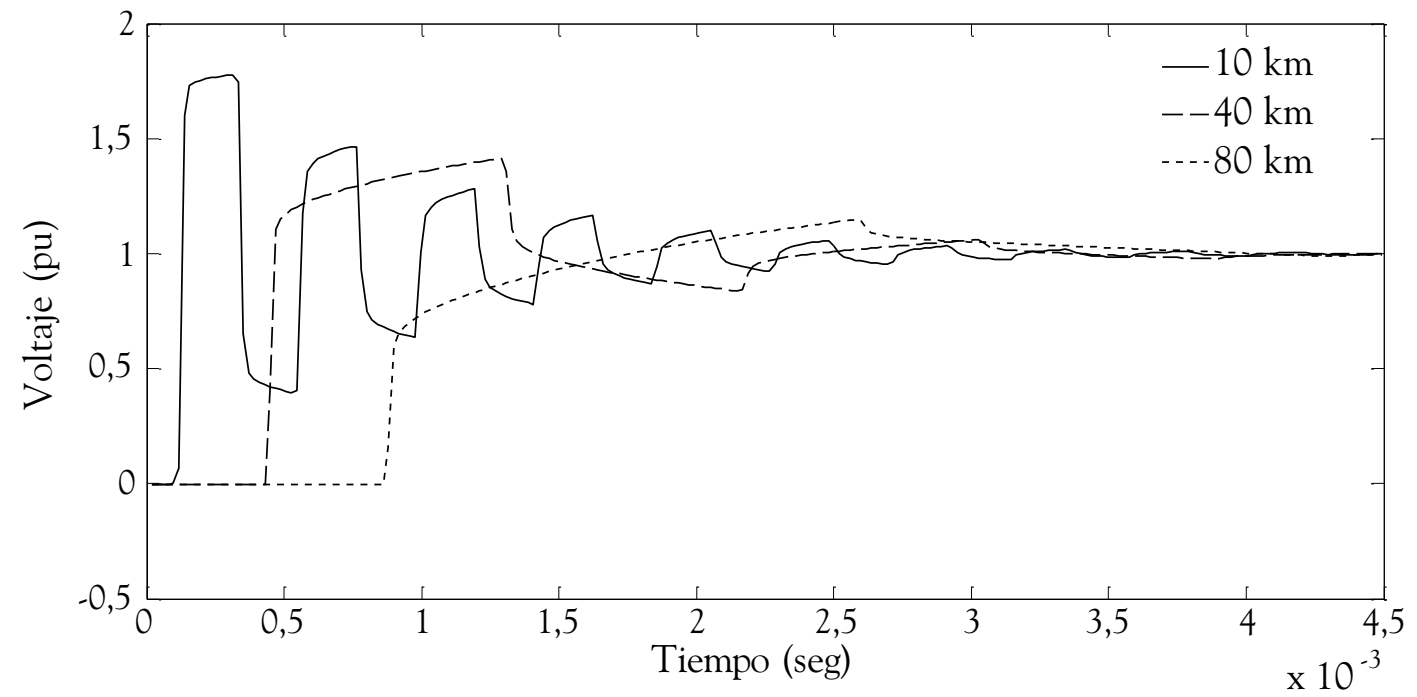

Figura 8. Respuesta transitoria del voltaje al final de la línea monofásica subterránea utilizando la formulación aproximada propuesta por Wedepohl y Wilcox [11]

Fuente: los autores

utilizar en el modelado y análisis de líneas de transmisión subterráneas. Es evidente que se obtendrán mejores respuestas transitorias en las líneas de transmisión con el modelo propuesto por Wedepohl y Wilcox [11], ya que tiene un error relativo menor que la formulación aproximada dada por la ecuación (11).

\section{Discusión y conclusiones}

Se ha presentado un análisis comparativo de las formulaciones aproximadas de impedancia interna para el estudio de transitorios de alta frecuencia en cables subterráneos. Se describieron los cables de alta tensión, así como el procedimiento para su modelado en estudios de propagación de pulsos electromagnéticos en cables subterráneos. El análisis se desarrolló en el dominio de la frecuencia y la metodología en general fue basada en la teoría de líneas de transmisión multiconductoras, 
análisis modal, teoría de redes de dos puertos y la transformada rápida de Fourier.

Se analizaron las formulaciones aproximadas de impedancia interna propuestas por Gary [17] y Wedepohl-Wilcox [11] para diferentes casos de estudio, en los que se evidenció que esta última formulación tiene un error menor tanto en valores reales como en valores imaginarios. Sin embargo, los resultados de las simulaciones demostraron que es posible utilizar cualquiera de las dos formulaciones aproximadas de impedancia interna, ya que se obtienen resultados aceptables y no se tiene una discrepancia notable en dichos resultados.

Es importante mencionar que la formulación aproximada de impedancia interna propuesta por Gary [17] es comúnmente utilizada en el modelado de líneas de transmisión aéreas; no obstante, de acuerdo con el análisis comparativo presentado en este artículo, es posible afirmar que dicha formulación aproximada puede ser también utilizada en el modelado y análisis de líneas de transmisión subterráneas.

Los resultados de las simulaciones de los casos de estudio mostraron que al aumentar la longitud de la línea monofásica subterránea se incrementa la impedancia de la misma, lo cual provoca una disminución en la magnitud del voltaje transitorio y un incremento del tiempo de desplazamiento de la onda de viaje.

\section{Referencias}

[1] J. Torres, "Análisis transitorio de redes de tierra en el dominio de la frecuencia”, Tesis de maestría, Instituto Tecnológico de Morelia, Morelia, Mich., 2004.

[2] F. Pollaczek, "Uber das feld einer unendlich langen wechsel stromdurchflossenen Einfachleitung". Electrishe Nachrichten Technik, Vol. 3, No. 9, pp. 339-360, 1926.

[3] L. V. Bewley, "Critique of Ground Wire Theory". AIEE Transactions, Vol. 50, No. 1, pp. 1-22, Mar., 1931.

[4] P. L. Bellaschi, "Impulse and 60-Cycle Characteristics of Driven Grounds". AIEE Transactions, Vol. 60, pp. 123-128, Mar., 1941.

[5] R. Rudenberg, "Fundamental Considerations on Ground Currents". Electrical Engineering, Vol. 64, pp. 1-13, Ene., 1945.

[6] E. D. Sunde, "Earth Conduction Effects in Transmission Systems”. Dover Publications Inc., Nueva York, 1968.
[7] L. M. Wedepohl y D. J. Wilcox, “Transient and Harmonic Induction in Underground Cables Systems”, Proc. IEE, Vol. 120, pp. 253-260, 1973.

[8] L. Marti, "Simulation of Transients in Underground Cables with Frequency Dependant Modal Transformation Matrices", IEEE Transactions on Power Delivery, Vol. 3, No. 3, pp. 1099-1110, 1988.

[9] N. S. Nahman, y D. R. Holt, "Transient analysis of coaxial cables using the skin effect approximation $A+B \sqrt{s}$ ", IEEE Transactions on Circuit Theory, Vol. 19, No. 5, pp. 443-451, 1972.

[10] A. Semlyen y A. Deri, "Time domain modeling of frequency dependent three phase transmission line impedance", IEEE Transactions on Power Apparatus and Systems, Vol. 104, No.6, pp. 1549-1555, 1985.

[11] L. M. Wedepohly D. J. Wilcox, “Transient analysis of underground power transmission systems: System-model and wave propagation characteristics", IEE Proceedings on Generation, Transmission and Distribution, Vol. 20, No. 2, pp. 253-260, 1973.

[12] S. Vujević, V. Boras y P. Sarajčev, "A novel algorithm for internal impedance computation of solid and tubular cylindrical conductors", International Review of Electrical Engineering (IREE), Vol. 4, No. 6, Part B, pp.1418-1425, 2009.

[13] W. Mingli y F. Yu, "Numerical calculations of internal impedance od solid and tubular cylindrical conductors under large parameters", IEE Proceedings on Generation, Transmission and Distribution, Vol. 151, No. 1, pp. 67-72, 2004.

[14] D. W. Knight, "Practical continuous functions and formulae for the internal impedance of cylindrical conductors". Version 2.04, Mar., 2010. [En línea]. Disponible en: http://www.g3ynh.info/zdocs/comps/Zint.pdf.

[15] J. Torres, A. I. Jardines y J. L. Guardado, "Análisis Transitorio de Cables Subterráneos". Memorias de la XI Reunión de Otoño de Potencia, Electrónica y Computación (ROPEC, 2009), Morelia, Mich., México, 2009, pp. 106-110.

[16] W. Johnson y M. Gram, High-Speed signal propagation: advanced black magic. Saddle River, NJ, Prentice-Hall, 2003, pp. 67-73.

[17] C. Gary, "Approche complete de la propagation multifilaire en haute frequence par utilization des matrices complexes", EDF Bulletin de la Direction des Etudes et Recherches, No. 3-4, pp. 5-20, 1976.

[18] F. A. Uribe, "Evaluación Algorítmica de la Integral de Pollaczek y sus aplicaciones en el Análisis de Transitorios Electromagnéticos en Sistemas de Transmisión Subterránea”, Tesis de doctorado, Centro de Investigación y de Estudios Avanzados del IPN, Guadalajara, Jal., México, 2002. 
[19] O. Saad, G. Gaba y M. Giroux, “A Closed-Form Approximation for Ground Return Impedance of Underground Cables", IEEe Transactions on Power Delivery, Vol. 11, No. 3, pp. 1536-1545, Jul., 1996.

[20] Unión Fenosa Internacional, "Conductores Desnudos de Aluminio con Alma de Acero (ACSR)". Anexo 2, 2004.
[21] F. A. Uribe, J. L. Naredo, P. Moreno y L. Guardado, “Electromagnetic Transients in Underground Transmission Systems Through the Numerical Laplace Transform". International Journal of Electrical Power and Energy Systems, Vol. 24, No. 3, pp. 215-221, Mar., 2002. 